\title{
Short-term efficacy of paraffin therapy and home-based exercise programs in the treatment of symptomatic hand osteoarthritis
}

\author{
Meliha Kasapoğlu Aksoy (i), ${ }^{1}$ Lale Altan (102 \\ ${ }^{1}$ Department of Physical Therapy and Rehabilitation, University of Health Sciences Bursa Yuksek Ihtisas Training and Research Hospital, Bursa, Turkey \\ ${ }^{2}$ Department of Physical Therapy and Rehabilitation, Uludağ University School of Medicine, Bursa, Turkey \\ Received: March 28, 2017 Accepted: July 27, 2017 Published online: October 02, 2017
}

\begin{abstract}
Objectives: This study aims to investigate the effects of a home-based exercise program in combination with paraffin therapy on pain, functional status, grip strength, and quality of life $(\mathrm{QoL})$ and to compare the outcomes of these two treatment modalities.

Patients and methods: This prospective, single-blind, randomized-controlled study included a total of 61 patients (8 males, 53 females, mean age 59.22 years; range, 35 to 78 years) who were diagnosed with hand osteoarthritis (HOA) according to the American College of Rheumatology criteria between November 2016 and February 2017. The first group (group 1) (n=31) received paraffin therapy + home-based exercise program and the second group (group 2) $(\mathrm{n}=30)$ received home-based exercise program alone. All patients were assessed using the Visual Analog Scale (VAS), Australian/Canadian (AUSCAN) Osteoarthritis Hand Index, Health Assessment Questionnaire (HAQ), Hand Grip Strength (HGS), and Finger Pinch Strength (FPS) at baseline and at two and six weeks after the intervention.

Results: In group 1, there were statistically significant improvements in all parameters at two and six weeks (p $<0.05)$. Statistically significant differences were observed in the HGS and AUSCAN Osteoarthritis Hand Index scores in group 2 at two and six weeks ( $\mathrm{p}<0.05)$. Inter-group analysis showed statistically significant differences in favor of group 1 in the VAS, HAQ, AUSCAN Osteoarthritis Hand Index, HGS, and FPS scores at two and six weeks compared to baseline scores $(\mathrm{p}<0.05)$.
\end{abstract}

Conclusion: Paraffin therapy combined with home-based exercise program in patients with symptomatic HOA provides effective pain control and is effective in improving functional status, HGS, FPS, and QoL in short term.

Keywords: Hand osteoarthritis; home-based exercise; paraffin therapy.

Osteoarthritis (OA) is the most common rheumatic disease in the population and its prevalence increases with age. ${ }^{[1]}$ Although the prevalence of hand osteoarthritis (HOA) may show variations, a recent study have reported a prevalence of $67 \%$ among females and $54.8 \%$ among males, as assessed by radiographic changes. ${ }^{[2]}$ Studies on HOA have reported $60 \%$ reduction in the hand grip strength (HGS). ${ }^{[3]}$ Significant pain, loss of range of motion, and reduced HGS associated with HOA, may considerably restrict the performance of daily living activities. ${ }^{[4,5]}$

Osteoarthritis management includes pharmacological and non-pharmacological treatment options. In particular, potential harmful effects of pharmacological agents should be taken into consideration in elderly patients with comorbidities, possibly taking multiple medications. ${ }^{[6]}$ Furthermore, non-pharmacological interventions including patient education, exercise, and local heat applications (i.e., paraffin, hot packs, and ultrasound) are widely recommended due to the unavailability of diseasemodifying drugs for OA. ${ }^{[6,7]}$ In the treatment of HOA, exercise and local applications of heat are also included in the treatment recommendations of the European League Against Rheumatism (EULAR). ${ }^{[7]}$

In a systemic review, a weak-level evidence for the association between paraffin therapy and pain relief, increased range of motion of the hand joints, and improved hand function in patients with HOA was reported. Despite the limited evidence for the efficacy of paraffin therapy, in vivo studies demonstrated that a temperature increase of $7.5^{\circ} \mathrm{C}$ in the joint capsule and

Corresponding author: Meliha Kasapoğlu Aksoy, MD. Sağlık Bilimleri Üniversitesi Bursa Yüksek İhtisas Eŭitim ve Araştırma Hastanesi, Fizik Tedavi ve Rehabilitasyon Kliniği, 16320 Ylldırım, Bursa, Turkey. e-mail: melihakasapoglu@hotmail.com 
a temperature increase of $4.5^{\circ} \mathrm{C}$ in the muscle occurred during the sessions of paraffin therapy. ${ }^{[8]}$ Local effects of paraffin therapy include relaxation of the muscle fibers and arterioles and vasodilation in the peripheral blood vessels, resulting in hyperemia, an increased transduction of tissue fluids, an increased lymph flow, and an increased absorption of exudates. ${ }^{[9,10]}$

Patients with HOA may benefit from strengthening the stabilizer muscles, range of motion exercises, and splinting. Patient education with exercise therapy has been also reported to be effective in the management of HOA. ${ }^{[1,12]}$ Although there are stronger evidences regarding positive effects of exercise on pain and functions in the studies on lower limb OA, there are limited, low-level evidence studies on the effects of exercises on HOA, and lower levels of evidence have been obtained by these studies. ${ }^{[7,11,12]}$

In the present study, we aimed to investigate the effects of a home-based exercise program in combination with paraffin therapy on pain, functional status, grip strength, and quality of life and to compare the outcomes of these two treatment modalities.

\section{PATIENTS AND METHODS}

This prospective, single-blind, randomizedcontrolled study was conducted in the Physical Therapy and Rehabilitation outpatient clinic of University of Health Sciences Bursa Training and Research Hospital, between November 2016 and February 2017. Patients who were diagnosed with HOA according to the American College of Rheumatology (ACR) criteria and had a Visual Analog Scale (VAS)-pain score of $\geq 4$ and aged between 35 and 75 years were included in this study. Exclusion criteria were as follows: carpal tunnel syndrome, De Quervain's tendinitis, tenosynovitis, Dupuytren's contracture, OA secondary to rheumatic diseases, OA secondary to a past fracture, open wounds and scars of the hand and wrist region, peripheral vascular diseases, prior vascular surgery of the hand, history of peripheral neuropathy, impaired sensory function of the hand, and cognitive impairment. Of a total of 70 patients screened, 61 (8 males, 53 females, mean age 59.22 years; range, 35 to 78 years) were eligible to the study.

Each patient underwent a radiographic assessment according to the Kellgren-Lawrence ${ }^{[13]}$ radiological scale. Only one hand, whichever caused a more pronounced pain, was included in the study. All patients were questioned about their complaints and underwent physical examination. This study was approved by the local Ethics Committee. All patients were informed about the study in detail and a written informed consent was obtained from each patient. The study was conducted in accordance with the principles of the Declaration of Helsinki.

The patients were randomized into two groups using a random number table. The first group (group 1) $(\mathrm{n}=31)$ received paraffin therapy + home-based exercise program and the second group (group 2$)(n=30)$ received home-based exercise program alone (Figure 1).

Group 1 patients underwent paraffin therapy over two weeks, five sessions a week, 10 sessions in total. All jewelry was removed from the hand before the session. The hand was dipped into paraffin with

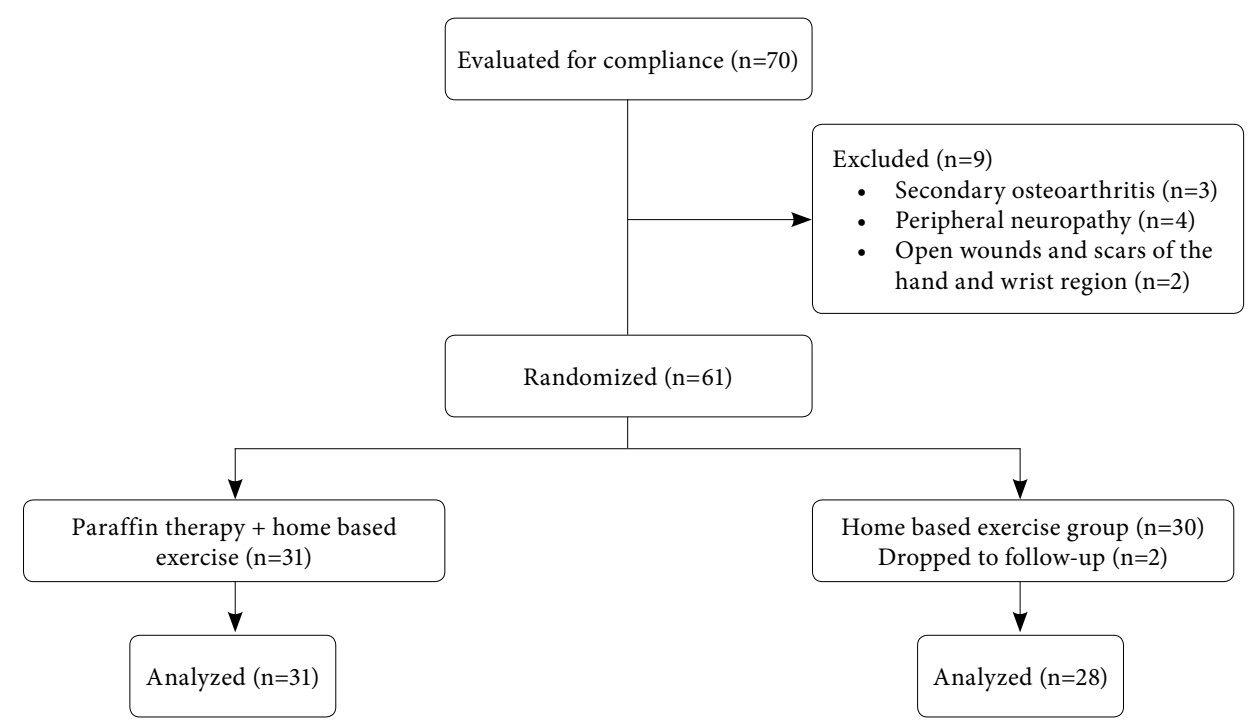

Figure 1. Flow diagram. 
fingers spread and the wrist in a neutral position and, then, the hand was wrapped in nylon and enveloped in a towel, unwrapped $20 \mathrm{~min}$ later and paraffin was peeled off the hand. Paraffin temperature was checked using a thermometer and adjusted to $52^{\circ} \mathrm{C}$. Paraffin therapy was administered along with the home-based exercise program and daily life activity recommendations. Exercise program included the hand grip strengthening, isometric exercises, and active resistance training.

Group 2 patients received home-based exercise program alone with daily life activity recommendations.

All patients were provided with exercise forms. The patients were assumed to exercise for 15 min twice a day and they were called up to check whether they adhered to the exercise program.

None of the patients in group 1 and group 2 received anti-inflammatory drugs (NSAIDs or paracetamol) in the treatment period or during follow-up.

Demographic characteristics of the patients were recorded. The Body Mass Index (BMI) was calculated as $\mathrm{kg} / \mathrm{m}^{2}$. The patients were assessed at baseline and at the end of two and six weeks after the intervention.

\section{Assessment tools}

1) Visual Analog Scale (VAS): The VAS was used for the assessment of pain. A $10 \mathrm{~cm}$ horizontal line was used for this purpose. ${ }^{[14]}$

2) Australian/Canadian (AUSCAN) Osteoarthritis Hand Index: The AUSCAN Osteoarthritis Hand Index is used to evaluate the functional state of the hand. It is a disease-specific questionnaire which assesses health status in three dimensions including pain (5 items), stiffness (1 item), and difficulty in the activities of daily living (9 items). The patients are asked to rate pain, stiffness, and disability they experience during the preceding 48 hours. All of the 15 items are rated on a scale of 0 to $4(0=$ not at all, $1=$ mild, $2=$ moderate, $3=$ severe, $4=$ extreme). The reliability and validity of AUSCAN Osteoarthritis Hand Index were shown previously. ${ }^{[15]}$

3) Health Assessment Questionnaire (HAQ): The HAQ has been developed for the assessment of patients with rheumatic diseases. It consists of 20 questions about eight activities (items); dressing, arising, eating, walking, hygiene, grip, and activities of daily living are questioned. Each answer is scored from 0 to 3 . The HAQ reflects the functional state of patients and this questionnaire has been shown to correlate with disease activity markers. ${ }^{[16]}$

4) Hand Grip Strength (HGS): A standard hand dynamometer (Jamar ${ }^{\circledR}$ Plus + Digital Hand Dynamometer from Patterson Medical by Sammons Preston, Bolingbrook, USA) was used to measure the grip strength. The reliability and validity of Jamar dynamometer is high; therefore, it has been considered gold standard in the assessment of grip strength. ${ }^{[17,18]}$ Grip strength tests were performed three times with one-min intervals and the mean of three measurements was calculated. The grip strength was measured in kilogram-force.

5) Finger Pinch Strength (FPS): The pinch strength was tested using a pinch meter $\left(\right.$ Jamar $^{\circledR}$ Pinch Gauge, TEC, Clifton, New Jersey, USA). Tip pinch was the type of pinch tested in the study and both hands were tested. The pinch meter was pinched between the tip of the thumb and the tip of the index finger to measure the pinch strength. The patients were asked to pinch as hard as they could. The test was repeated three times and the mean of three measurements was recorded in kilogram. The reliability and validity of the pinch strength test were shown by Mathiowetz et al. ${ }^{[19]}$

Table 1. Baseline demographic characteristics of patients

\begin{tabular}{|c|c|c|c|c|c|c|c|c|c|c|c|}
\hline & \multicolumn{5}{|c|}{ Group $1(\mathrm{n}=31)$} & \multicolumn{5}{|c|}{ Group $2(\mathrm{n}=28)$} & \multirow[b]{2}{*}{$p$} \\
\hline & $\mathrm{n}$ & $\%$ & Mean $\pm S D$ & Median & Min-Max & $\mathrm{n}$ & $\%$ & Mean \pm SD & Median & Min-Max & \\
\hline Age (year) & & & $57.2 \pm 10.6$ & & & & & $61.3 \pm 8.4$ & & & 0.130 \\
\hline Sex & & & & & & & & & & & 1.000 \\
\hline Female & 28 & 90.3 & & & & 25 & 89.3 & & & & \\
\hline Male & 3 & 9.7 & & & & 3 & 10.7 & & & & \\
\hline Visual Analog Scale & & & & 7.16 & $4-10$ & & & & 6.57 & $5-8$ & 0.105 \\
\hline $\operatorname{BMI}\left(\mathrm{kg} / \mathrm{m}^{2}\right)$ & & & & 29.18 & $19.0-4$ & & & & 29.46 & $25.6-35.2$ & 0.855 \\
\hline HAQ & & & & 0.80 & $0.2-1.8$ & & & & 0.60 & $0.2-1.5$ & 0.130 \\
\hline \multicolumn{12}{|l|}{ AUSCAN } \\
\hline Osteoarthritis Hand Index & & & & 27 & $23-3$ & & & & 26 & $24-30$ & 0.168 \\
\hline Hand grip strength (kg) & & & & 48.77 & $21.1-83.6$ & & & & 42.03 & $23.4-58.3$ & 0.109 \\
\hline Pinch strength (kg) & & & & 12.25 & $6-20$ & & & & 11.10 & $7-15$ & 0.204 \\
\hline
\end{tabular}

SD: Standard deviation; Min: Minimum; Max: Maximum; BMI: Body Mass Index; HAQ: Health Assessment Questionnaire; AUSCAN: Australian/Canadian Osteoarthritis Hand Index; $\mathrm{p}<0.05$ statistically significant; Fisher's exact test and Mann-Whitney $\mathrm{U}$ test were used for inter-group comparisons. 


\section{Statistical analysis}

Statistical analysis was performed using the IBMSPSS version 22.0 statistical software (IBM Corp., Armonk, NY, USA). For categorical variables, the Fisher's exact test was used to assess the differences between two sexes. Descriptive data were expressed in mean and standard deviation (SD), median (min$\max )$ values, and percentage. The Shapiro-Wilk test was used to assess whether the data were normally distributed. The independent samples t-test was used for inter-group comparisons, when the variables were found to be normally distributed. For abnormally distributed data, the Wilcoxon signed-rank test was used for intra-group comparisons, while the Mann-Whitney $U$ test was used for inter-group comparisons. A $p$ value of $<0.05$ was considered statistically significant.

\section{Power analysis}

$G^{\star}$ Power 3.0.10 software package (Franz Faul, Christian-Albrechts-Universität, Kiel, Germany) was used for the power analysis. The study power $(1-\beta)$ was found to be 0.84 with post-hoc $n 1=31, \mathrm{n} 2=28, \alpha=0.05$, Effect size $d=0.8$. The effect size was calculated using the VAS scores. As the numbers of two groups were not equal, the independent $\mathrm{t}$-test was used for the VAS scores and $\mathrm{F}$ value $=8.709$ was obtained. The calculated effect size was 0.8 for this study.

\section{RESULTS}

Of 61 patients, two were lost to follow-up in group 2 and 31 patients in group 1 and 28 patients in group 2 completed the study.
There was no statistically significant differences in the mean age, sex, BMI, disease duration, radiological grade, baseline VAS, HAQ, AUSCAN Osteoarthritis Hand Index, FPS, and HGS scores between the groups $(\mathrm{p}<0.05)$ (Table 1).

However, in group 1, statistically significant improvements were observed in all parameters assessed at two and six weeks $(\mathrm{p}<0.05)$ (Table 2). There were also statistically significant differences in the HGS and AUSCAN Osteoarthritis Hand Index scores in group 2 at two and six weeks $(\mathrm{p}<0.05)$.

In addition, inter-group comparisons revealed a statistically significant difference in favor of group 1 in the VAS, HAQ, AUSCAN Osteoarthritis Hand Index, HGS, and FPS scores at two and six weeks, compared to the baseline scores $(\mathrm{p}<0.05)$ (Table 3$)$.

\section{DISCUSSION}

In this study, paraffin therapy in combination with home-based exercise program provided significant improvements in pain scores and functional activity, while significant improvements were observed in the HGS and functional scores in the patients who received exercise alone.

Local applications of heat can be used in the management of $\mathrm{OA}$ as an adjuvant non-pharmacological treatment modality. ${ }^{[6,7]}$ Local applications of heat are also used in the treatment of HOA in combination with other treatment modalities and they are used to relieve muscle spasms and have sedative effects, in particular. $^{[20]}$ Furthermore, local applications of heat

Table 2. Intra-group comparison of post-treatment and six-week values

\begin{tabular}{|c|c|c|c|c|c|c|}
\hline & \multicolumn{2}{|c|}{ After-treatment } & \multicolumn{2}{|c|}{$6^{\text {th }}$ week } & \multicolumn{2}{|c|}{$p$} \\
\hline & Median & Min-Max & Median & Min-Max & W0-W2 & W0-W6 \\
\hline \multicolumn{7}{|l|}{ Visual Analog Scale } \\
\hline Group $1(\mathrm{n}=31)$ & 4.38 & $0-8$ & 4.16 & $1-8$ & 0.000 & 0.000 \\
\hline Group $2(\mathrm{n}=28)$ & 6.17 & $1-9$ & 5.96 & $1-9$ & 0.187 & 0.062 \\
\hline \multicolumn{7}{|c|}{ Health Assessment Questionnaire } \\
\hline Group $1(n=31)$ & 0.35 & $0-1.45$ & 0.30 & $0.1-1.1$ & 0.000 & 0.000 \\
\hline Group $2(n=28)$ & 0.62 & $0.3-1.3$ & 0.55 & $0.1-1.5$ & 0.419 & 0.648 \\
\hline \multicolumn{7}{|c|}{ AUSCAN Osteoarthritis Hand Index } \\
\hline Group $1(\mathrm{n}=31)$ & 23 & $0-8$ & 23 & $20-29$ & 0.000 & 0.000 \\
\hline Group $2(\mathrm{n}=28)$ & 26 & $22-29$ & 25.50 & $21-30$ & 0.012 & 0.005 \\
\hline \multicolumn{7}{|c|}{ Hand Grip Strength (kg) } \\
\hline Group $1(n=31)$ & 51.05 & $21-84$ & 50.73 & $20-83$ & 0.000 & 0.000 \\
\hline Group $2(\mathrm{n}=28)$ & 42.71 & $23.6-59$ & 42.48 & $26-59$ & 0.001 & 0.015 \\
\hline \multicolumn{7}{|l|}{ Pinch Strength $(\mathrm{kg})$} \\
\hline Group $1(\mathrm{n}=31)$ & 13.70 & $9-22$ & 13.35 & $8-22$ & 0.000 & 0.001 \\
\hline Group $2(n=28)$ & 11.25 & $8-15$ & 11.32 & $8-15$ & 0.415 & 0.134 \\
\hline
\end{tabular}


Table 3. Comparison of scores between groups

\begin{tabular}{|c|c|c|c|c|c|c|c|c|c|c|}
\hline & \multicolumn{5}{|c|}{ After-treatment } & \multicolumn{5}{|c|}{$6^{\text {th }}$ week } \\
\hline & \multicolumn{2}{|c|}{ Group 1} & \multicolumn{2}{|c|}{ Group 2} & \multirow[b]{2}{*}{$p$} & \multicolumn{2}{|c|}{ Group 1} & \multicolumn{2}{|c|}{ Group 2} & \multirow[b]{2}{*}{$p$} \\
\hline & Median & Min-Max & Median & Min-Max & & Median & Min-Max & Median & Min-Max & \\
\hline VAS & -2.77 & $-9-1$ & -0.39 & $-6-2$ & $<0.001$ & -3.00 & $-9-1$ & -0.60 & $-4-3$ & $<0.001$ \\
\hline HAQ & -0.4 & $-1.1--0.1$ & -0.025 & $-0.5-0.2$ & $<0.001$ & -0.45 & $-1.25-0$ & 0.00 & $-0.6-0.2$ & $<0.001$ \\
\hline \multicolumn{11}{|l|}{ AUSCAN } \\
\hline Osteoarthritis Hand Index & -4 & $-7--1$ & 0.00 & $-5-2$ & $<0.001$ & -4 & $-6-0$ & -1.0 & $-5-2$ & $<0.001$ \\
\hline HGS (kg) & 2.28 & $-0.8-9.6$ & 0.67 & $-2.8-8.8$ & $<0.001$ & 1.96 & $-1.1-9.6$ & 0.45 & $-4-3.6$ & $=0.026$ \\
\hline PS (kg) & 1.45 & $-3-6$ & 0.14 & $-2-2$ & $<0.001$ & 1.09 & $-2-5.00$ & 0.21 & $-1-2$ & $=0.012$ \\
\hline
\end{tabular}

Min: Minimum; Max: Maximum; VAS: Visual Analog Scale; HAQ: Health Assessment Questionnaire; AUSCAN: Australian/Canadian Osteoarthritis Hand Index; HGS: Hand Grip Strength; PS: Pinch Strength; $\mathrm{p}<0.05$ statistically significant; The Mann-Whitney U test was used for inter-group comparisons.

reduce peripheral nerve conduction velocities, increase peripheral endorphin production, and raise the pain threshold. ${ }^{[21]}$ As suggested by the gate control theory which was developed by Melzack and Wall, ${ }^{[21]}$ thermal stimuli may block pain transmission by acting as peripheral stimuli. Superficial heat exerts its therapeutic effects by providing analgesia, hyperemia, and changing local or systemic temperature and reducing the muscle tone. Therapeutic effects associated with these physiological effects include pain and muscle spasm relief, and an increased metabolism..$^{[22]}$

In a review on the efficacy of paraffin therapy in patients with rheumatoid arthritis (RA), significant improvements were observed in the hand functions of patients with RA who were treated with paraffin therapy in combination with exercise. ${ }^{[23]}$ In consistent with this study, Dellhag et al. ${ }^{[2]}$ reported that paraffin therapy used in combination with an exercise program was more effective than an exercise program alone in patients with RA. Furthermore, in a study including patients with scleroderma, one hand of the patient was treated with paraffin plus exercise, while the other hand was treated with exercise alone, and combination therapy with paraffin plus exercise was found to increase mobility, reduce stiffness, and increase elasticity. ${ }^{[25]}$ In another study, paraffin therapy combined with joint mobilization techniques was found to be more effective than the joint mobilization techniques alone in the management of stiff hand syndrome. ${ }^{[26]}$ Only patients with HOA were included in our study and HOA is known to have a better course than that of RA, scleroderma, or stiff hand syndrome. ${ }^{[27-29]}$ At the end of study, we observed significant improvements in pain, hand functions, and daily living activities in patients with HOA who were treated with paraffin therapy plus exercise.

In a study evaluating the efficacy of paraffin therapy in the management of HOA, significant improvements were observed in daily living activities,
VAS scores at rest and AUSCAN Osteoarthritis Hand Index scores compared to the control group. ${ }^{[30]}$ In addition, compared to the control group, significant improvements were observed in the HGS and FGS in the paraffin group. In consistent with these studies, we found significant improvements in the VAS, AUSCAN Osteoarthritis Hand Index, and HAQ scores and significant increases in the HGS and FGS scores in the patients receiving paraffin + home-based exercise. We also found statistically significant differences in the HGS and AUSCAN Osteoarthritis Hand Index scores in these patients, compared to those who received exercise alone.

Increased prevalence rates of HOA in elderly and a higher incidence of concurrent chronic systemic diseases in this population take local treatments such as paraffin therapy to forefront, particularly when considering drawbacks of medical treatments. In addition, the only evidence-based, non-pharmacological treatment of HOA is exercise programs with patient education. ${ }^{[7]}$ In our study, we also found significant increases in the HGS scores in patients who were treated with homebased exercise program alone including protective and strengthening exercises.

On the other hand, this study was unable to be designed as a double-bind, controlled study, due to the impossibility of blinding the patients to paraffin therapy that they received. Therefore, a sham effect could not be excluded. However, the physiatrist who made these assessments could be blinded.

In conclusion, in this study, paraffin therapy combined with home-based exercise program provided effective pain control and was found to be effective in improving functional status, HGS, FGS, and quality of life. Furthermore, at the end of study, improvements were sustained during follow-up until the end of the study. Based on these findings, the addition of paraffin therapy and home-based exercises to conventional treatments of HOA may further improve hand 
functions and provide a better pain relief. However, further, large-scale studies are needed to conclude.

\section{Declaration of conflicting interests}

The authors declared no conflicts of interest with respect to the authorship and/or publication of this article.

\section{Funding}

The authors received no financial support for the research and/or authorship of this article.

\section{REFERENCES}

1. Zhang Y, Niu J, Kelly-Hayes M, Chaisson CE, Aliabadi P, Felson DT. Prevalence of symptomatic hand osteoarthritis and its impact on functional status among the elderly: The Framingham Study. Am J Epidemiol 2002;156:1021-7.

2. Dahaghin S, Bierma-Zeinstra SM, Ginai AZ, Pols HA, Hazes JM, Koes BW. Prevalence and pattern of radiographic hand osteoarthritis and association with pain and disability (the Rotterdam study). Ann Rheum Dis 2005;64:682-7.

3. Kjeken I, Dagfinrud H, Slatkowsky-Christensen B, Mowinckel P, Uhlig T, Kvien TK, et al. Activity limitations and participation restrictions in women with hand osteoarthritis: patients' descriptions and associations between dimensions of functioning. Ann Rheum Dis 2005;64:1633-8.

4. Zhang W, Doherty M, Leeb BF, Alekseeva L, Arden NK, Bijlsma JW, et al. EULAR evidence-based recommendations for the diagnosis of hand osteoarthritis: report of a task force of ESCISIT. Ann Rheum Dis 2009;68:8-17.

5. Bagis S, Sahin G, Yapici Y, Cimen OB, Erdogan C. The effect of hand osteoarthritis on grip and pinch strength and hand function in postmenopausal women. Clin Rheumatol 2003;22:420-4.

6. Rannou F, Poiraudeau S. Non-pharmacological approaches for the treatment of osteoarthritis. Best Pract Res Clin Rheumatol 2010;24:93-106.

7. Zhang W, Doherty M, Leeb BF, Alekseeva L, Arden NK, Bijlsma JW, et al. EULAR evidence based recommendations for the management of hand osteoarthritis: report of a Task Force of the EULAR Standing Committee for International Clinical Studies Including Therapeutics (ESCISIT). Ann Rheum Dis 2007;66:377-88.

8. Borrell RM, Parker R, Henley EJ, Masley D, Repinecz M. Comparison of in vivo temperatures produced by hydrotherapy, paraffin wax treatment, and Fluidotherapy. Phys Ther 1980;60:1273-6.

9. Myrer JW, Johnson AW, Mitchell UH, Measom GJ, Fellingham GW. Topical analgesic added to paraffin enhances paraffin bath treatment of individuals with hand osteoarthritis. Disabil Rehabil 2011;33:467-74.

10. Stimson CW, Rose GB, Nelson PA. Paraffin bath as thermotherapy: an evaluation. Arch Phys Med Rehabil 1958;39:219-27.

11. Rannou F, Poiraudeau S. Non-pharmacological approaches for the treatment of osteoarthritis. Best Pract Res Clin Rheumatol 2010;24:93-106.
12. Valdes K, Marik T. A systematic review of conservative interventions for osteoarthritis of the hand. J Hand Ther 2010;23:334-50.

13. Kellgren JH, Lawrence JS. Radiological assessment of osteoarthrosis. Ann Rheum Dis 1957;16:494-502.

14. Price DD, McGrath PA, Rafii A, Buckingham B. The validation of visual analogue scales as ratio scale measures for chronic and experimental pain. Pain 1983;17:45-56.

15. Bellamy N, Campbell J, Haraoui B, Buchbinder R, Hobby K, Roth JH, et al. Dimensionality and clinical importance of pain and disability in hand osteoarthritis: Development of the Australian/Canadian (AUSCAN) Osteoarthritis Hand Index. Osteoarthritis Cartilage 2002;10:855-62.

16. Küçükdeveci AA, Sahin H, Ataman S, Griffiths B, Tennant A. Issues in cross-cultural validity: example from the adaptation, reliability, and validity testing of a Turkish version of the Stanford Health Assessment Questionnaire. Arthritis Rheum 2004;51:14-9.

17. Mathiowetz V. Comparison of Rolyan and Jamar dynamometers for measuring grip strength. Occup Ther Int 2002;9:201-9.

18. Shechtman O, Gestewitz L, Kimble C. Reliability and validity of the DynEx dynamometer. J Hand Ther 2005;18:339-47.

19. Mathiowetz V, Weber K, Volland G, Kashman N. Reliability and validity of grip and pinch strength evaluations. J Hand Surg Am 1984;9:222-6.

20. Conaghan PG, Dickson J, Grant RL. Care and management of osteoarthritis in adults: summary of NICE guidance. BMJ 2008;336:502-3.

21. Melzack R, Wall P. Pain mechanisms: A new theory. Science 1965;150:971-9.

22. Bender T, Karagülle Z, Bálint GP, Gutenbrunner C, Bálint PV, Sukenik S. Hydrotherapy, balneotherapy, and spa treatment in pain management. Rheumatol Int 2005;25:220-4.

23. Ayling J, Marks R. Efficacy of paraffin wax baths for rheumatoid arthritic hands. Physiotherapy 2000;86:190-201.

24. Dellhag B, Wollersjö I, Bjelle A. Effect of active hand exercise and wax bath treatment in rheumatoid arthritis patients. Arthritis Care Res 1992;5:87-92.

25. Sandqvist G, Akesson A, Eklund M. Evaluation of paraffin bath treatment in patients with systemic sclerosis. Disabil Rehabil 2004;26:981-7.

26. Sibtain F, Khan A, Shakil-Ur-Rehman S. Efficacy of paraffin wax bath with and without joint mobilization techniques in rehabilitation of post-traumatic stiff hand. Pak J Med Sci 2013;29:647-50.

27. Sandqvist G, Akesson A, Eklund M. Evaluation of paraffin bath treatment in patients with systemic sclerosis. Disabil Rehabil 2004;26:981-7.

28. Fumagalli M, Sarzi-Puttini P, Atzeni F. Hand osteoarthritis. Semin Arthritis Rheum 2005;34(6 Suppl 2):47-52.

29. Jonsson B, Larsson SE. Hand function and total locomotion status in rheumatoid arthritis. An epidemiologic study. Acta Orthop Scand 1990;61:339-43.

30. Dilek B, Gözüm M, Şahin E, Baydar M, Ergör G, El O, et al. Efficacy of paraffin bath therapy in hand osteoarthritis: a single-blinded randomized controlled trial. Arch Phys Med Rehabil 2013;94:642-9. 